\title{
Coding of nonsense vs the detection of patterns
}

\author{
FRANK RESTLE \\ Indiana University, Bloomington, Indiana 47405
}

\begin{abstract}
Two theories are contrasted: coding information into arbitrary characters vs extracting patterns from a flux of stimulation. It is argued that coding is the process people use to memorize nonsense and, for that reason, is a poor model for the applied psychology of learning. The contrasting idea, that Ss perceive patterns and extract information, can be used to improve education by leading teachers to organize their material. It is also argued that most demonstrations of coding can be interpreted as examples of pattern perception.
\end{abstract}

Since I am not a coding theorist, my first response, naturally enough, is to consider the precise meaning of the term "coding." If one comes to a term late, it is important to become clear as to its proper use, so as not to announce one's arrival on the scene with a pratfall. Where does the word coding come from and what does it mean?

There are two main meanings in the dictionary: (1) as a code of law, meaning an organized body of laws or rules; (2) a system of signals, characters, or words with arbitrary meanings, used to communicate intelligence. Since cognitive psychologists speak of encoding and decoding, which relate to deciphering, and never mention codifying; which would relate to a code of laws, I am confident that coding is used in the sense of a system of arbitrary characters used in communication.

As the term is normally used, a code is an arbitrary set of symbols. Any message can be written in the cipher system and decoded to its original form. In fact, in a proper code, there is no problem to encoding and decoding arbitrary materials, including perfect nonsense, and nonsense materials might well be used as a test of the system.

This is the nature of a code. It is capable of expressing a certain number of things. Any message can be coded and, usually, decoded back to the original message. Nonsense can be coded just as well as sense, for coding does not respond to the meaning of the message but only to the symbols used.

\section{CODING IN COGNITIVE PSYCHOLOGY}

Coding is invoked at four levels in cognitive psychology; at the level of perception, at the level of transfer from the sensory buffer to short-term memory, at the transfer from short-term memory to long-term memory, and at retrieval from memory. At each of these points, there is a serious question as to the meaning of the coding process and an issue as to whether "coding" is the best representation.

At the sensory level, we have the transformation from physical energy, through response of sense organs, into neural impulses. An example is the encoding of tastes into different combinations of three elements or the selection by the cat's visual cortex of spots, lines, borders, and so forth, as worked out by Hubel and Wiesel (1959). Presumably, this sensory operation may be thought of as encoding the stimulus into a set of categories or into a list of attributes. The process is encoding in that the particular neurons used are arbitrary, and the message is fit into a set of properties determined in advance.

There is a clear issue with respect to sensory coding, and it is put before us most energetically by the Gibsons; I shall use E. J. Gibson's (1969) book as my main source. They argue that coding cannot be arbitrary but must consist of selecting from the environment properties and relationships that are already there. There must be an edge in the visual field. Given that edge, the cognitive system can completely ignore it, can lump it together with some other properties of the field, or can single it out as a significant property. What is important, say the Gibsons, is the existence of the property in the outside world and then the system selectively sensitive to that property.

The opposing position in effect says that the main categories are developed within the organism (Bruner, 1964). Incoming stimuli, even if they are only partial or approximate exemplars of the categories, become able to determine the meaningful coded response.

An example of the strongest form of the coding hypothesis is the motor theory of speech perception (Liberman, Cooper, Shankweiler, \& Studdert-Kennedy, 1967), which says that acoustic input is fit into a relatively simple and rigid set of categories. In some cases, according to Liberman, there simply is no constant acoustic element to account for a phoneme, and instead we select and recognize $/ \mathrm{m} /$ by the system that generates the sound. In other words, we understand (encode) speech sounds by a process of analysis by synthesis (Stevens \& Halle, 1967), and the synthesis system is primary. The speech code is categorical and arbitrary and contrasts with the continuous acoustical signal upon which it operates.

The alternative argument must be that the phonemes are not low-order stimulus properties but more complex sound combinations and that the properties that arrange these phonemes and permit their discrimination correspond closely to the properties that permit speaking them. It is apparently undisputed that a deaf 
child, despite the most careful instruction and training in articulation, cannot make sounds that closely mimic the speech of hearing people. It is apparently the constant interaction of speaking and hearing one's speech that leads to the ability to communicate in language. This suggests that speech is not an arbitrary motar-dominated code but instead must be a system of discriminations that can serve, in common, the hearing and the speaking systems.

What I propose to do is to press this issue farther on and criticize the other uses of the concept of a cognitive code in about the way the Gibsons criticize the users of the idea of sensory coding. Having already dealt with sensory or perceptual processing, I have three stages left to deal with the usual experiments: taking information from perception (the sensory buffer) into primary memory or short-term store; taking information from primary to secondary memory, or into long-term store; and retrieval of information from wherever it may be, presumably mainly in long-term store. Each of these stages of processing might involve coding and changes of code, though, of course, the process seems to become rather unwieldy if the same information changes form four times before it can be used.

At each point of the discussion, I have two questions to ask: First, does the $S$ really encode information into an arbitrary system of characters or is he just selecting from the information whatever he needs? Second, if the $\mathrm{S}$ is using an arbitrary code, what does this signify about the situation; does it mean, as I suspect, that we have given the $\mathrm{S}$ nonsense from which no meaningful information can be extracted and forced him to memorize it? If so, then coding may be only a model of bad educational practice, blind memorizing, and the opposite of true cognition.

First, how does information get into short-term store? In many experiments, the information is presented visually in the form of written letters or words. As Broadbent has pointed out, when information comes in at a high rate, either acoustic or visual, some of it will be lost, and the person can be quite selective about what he loses. If the $\mathrm{S}$ is reading letters, he must recognize them, and this recognition and the subsequent retention are referred to as short-term store.

Posner and Mitchell (1967) have made it clear that people can match two capital As rapidly, and, when they must match capital $\mathbf{A}$ and lowercase a, which look different but have the same name, they are delayed by a time interval that can be attributed to "encoding." That is, when comparing capital As, a "visual" comparison is possible, but when capital and lowercase must be compared, the $S$ must first translate each visual shape into its name, an articulatory-auditory code. Since both have the same name, they can then be identified.

Is this result really evidence for "encoding"? In comparing two figures with the same visual appearance, the $\mathrm{S}$ can use a variety of features, such as overall shape, that may be rapidly scanned. When capital and lowercase must be compared. it is necessary to use the specific system of distinctive features of the "reading" system. This system is quite slow when individual unpredictable letters must be read. When a letter has been identified by the necessary system of critical features, it can be named and thereby realized within the articulatory-acoustical system. My point is that the $\mathbf{S}$ has no reason to read it aloud, once he has identified it and extracted the necessary information.

When E.J. Gibson approaches the question of reading. she thinks of the written language as a system of graphemic rules, the spoken language as a somewhat related system of phonemic rules, and "reading" as the process of going from one system to the other-a straightforward problem in coding and decoding. In the mechanical sense of the verb "to read," the task is to articulate in spoken form the literal message that is given as a written text.

That is all "reading" can mean in experiments using isolated letters or nonsense syllables, and it is almost all that "reading" can mean in the letter by letter and word by word experiments usually done. However, in a study such as Harry Levin's work on the "eye-voice-span," we find that the adult reader takes in quite a mass of information visually, and then puts it out verbally as a separate, differently organized operation (Levin \& Turner, 1966). This gets a little closer to real reading, the extraction of information from a written text, in which direct eye-voice translation is not required at all.

I propose that, if there is a meaningful and organized pattern in the written material, a system of rules and a structure to be detected, then reading is best understood as extraction of that pattern. The primary problem is not to put that information into another code, that of spoken English; it is merely to extract it.

I think that psychologists have, in some cases, been so enamoured of the "coding" hypothesis that they have accepted it wrongly. My favorite example is the well known study by Glanzer and Clark (1963), in which they employ what is called the "verbal-loop hypothesis." They presented Ss with a string of eight binary events that were to be reproduced after brief exposure. One string, for example, 00000000 , was very easy to handle and another simple alternation, 01010101, was also easy. A disorderly string, like 10011101 , might be quite difficult to handle. Glanzer and Clark tried several ways to predict the difficulty of these strings from information theory and such ideas, but none of their efforts was highly successful. They had Ss try to describe these sequences and found that 00000000 was described as "all zeros," 01010101 was described as "alternating 0 and 1," and 10011101 received a long, item by item description. Somehow this led them to the idea that it was the length of the verbal code that made some items more difficult than others. That is, if the string required a long description, then Ss used a long description but would make errors in forming it, or would forget it, or would make errors in generating their reproduction of it. 
If the string had a short description, the $S$ had no difficulty remembering and handling this easy coded form.

I cannot disprove this hypothesis, exactly, but I find it rather strange. The fact is that 00000000 can be generated from a very simple set of rules because it embodies a very simple pattern, requiring only the operation of repetition to produce it. 01010101 is slightly more complex, since it requires repetition of the larger structure $(01)$, which is itself an alternation. The string 10011101 is much more difficult to generate from any coherent system of rules, since it does not have a simple pattern. A $S$ engaged in pattern detection will be turned away by the string 10011101 by his failure to find a pattern. The fact that the string is nonsense means that the description will be long and uninteresting and probably difficult for the S to remember. The S's basic problem is pattern perception, extracting the information from the string in a form that can be used to reconstruct it. Therefore, incoherence of the string produces both the long disorganized verbal code and the difficulty in remembering the string. More recent work by Vitz and Todd (1969) and in my laboratory (Restle, 1970; Restle \& Brown, 1970; Restle \& Burnside, 1972) has shown that you can get a very precise picture of pattern perception from the study of patterned strings and how their patterns are extracted, and that the verbal loop hypothesis merely gives us an incidental sideways look at the problem.

Information is often said to be recoded when it goes from short-term to long-term memory. Bower's interesting idea is that the information is encoded in fairly uneconomical form in short-term memory but that a search is performed to find corresponding information in long-term memory. If any segment of short-term memory can be matched with something in long-term store, the whole segment is replaced in STM with a token from LTS. In this way, a large bulk of code is replaced by a very short indication of what it is. Of course, it is also possible to replace XFZ with "extremely fat zebra," which replaces a small amount of code with a larger but more memorable block of code. However, if the extremely fat zebra arouses a unified image and that image can somehow be tagged in a unique, systematic way, then perhaps the whole recording operation is successful. I think this is about the way Bower intends to develop his theory of mnemonic devices.

In recent books, Restle and Greeno (1970) and Kintsch (1970) emphasize the functional requirements of coding for long-term memory. That is, if an item is not coded differentially, there is no way it can be recalled differentially when needed. Furthermore, when a relatively long list of material is to be learned, separate and unique coding for each item may place a heavy load upon the $S$, who would be much better off to integrate the separate code into one unified system. This view. put forward by Greeno, is supported by the finding that Ss change the codes they use in remembering paired-associate problems during periods of overtraining. In this general problem, Kintsch reviews the earlier findings that semantic cues are more important than acoustical cues for storing information in long-term memory.

The Greeno-Kintsch-Restle model can be traced back to work in discrimination learning, and the "code" can be related almost directly to the "cue" of discrimination learning. These theories say that a $\mathrm{S}$ must extract unique information from each item in the list in order to remember it differentially. Whether he also "encodes" this information into another system is quite unknown. It seems to me that Greeno's result is that Ss extract more information from an item with overtraining than they do just getting to criterion. That sounds plausible. Kintsch"s finding that semantic "codes" are used for long-term memory seems to agree in a natural way with the finding that meaningful material is memorable, so it is natural that Ss who must remember for a long time will attempt to extract not superficial appearance cues or sounds, but meanings. Beyond this, the "code" is usually a figment of the imagination, sometimes of the E's imagination.

Thus, at every stage of information processing, current theory is likely to invoke "coding," but the actual results obtained are mainly evidence about the information extracted. Bower's work on mnemonic devices is an exception, and here, I believe, the fact is that the $S$ is required to try to memorize nonsense material and can do so by encoding it into another organized mnemonic system. Similarly, when Conrad forced Ss to recognize and remember too many disorganized letters, they encoded the visual form into auditory-acoustical names and then made memory errors with these names. Again, a difficult and unpatterned task was used, and Ss attempted to solve it by recoding.

A scene, situation, or sequence of events may express a pattern, if the various details can be generated by systematic application of rules. Psychologists have been surprisingly unsuccessful at determining what rules our Ss are likely to look for and at finding ways to construct materials that do and do not conform to such rules. Instead, we have bent our efforts to randomizing, counterbalancing, disguising, homogenizing, and generally disorganizing the materials we give our Ss. In most experiments in verbal learning and information processing, we destroy the information completely. Some Ss then "recode," putting the material in other forms so as to try to detect a hidden pattern. For example, they may read letters aloud in the hope that their sounds may rhyme, form a rhythmic pattern. or sound like something recognizable and intelligible.

Faced with nonsense, a $S$ can remember material without being able to find any complete pattern in it This is the problem solved by a mnemonic system: the $\mathrm{S}$ supplies a system, unrelated to what he is trying to leam. but to which he can hook any arbitrary. disorganized 
mess of nonsense given him. This is the most extreme case of blind memorizing, as opposed to true learning.

Suppose we give our Ss material that is not perfectly nonsensical but has some constraints within it and, therefore. consists of broken or badly damaged patterns. That is. most of the material in such a list can be generated from a coherent system of rules, but there are enough exceptions to keep the learner dissatisfied. In this case, it appears that Ss tend to detect the rules and try using them, and perhaps then systematically have difficulty with items that deviate from the general rule they are trying to use. This could be thought of as learning supported by memorizing.

Finally, as we approach the learning of meaningful and organized material, we find that the main process is one of detecting the pattern. The material is not turned into some foreign code with its own arbitrary rules; a more apt description is that the pattern existing in the material is found and its rules are educed. I think that Ss are severely limited in their ability to find such patterns, in that they have great difficulty picking out unfamiliar or unexpected patterns. However, as we know, organized material if so learned is well remembered, probably for the healthy lifetime of the learner. Access is remarkably rapid and complete. Finally, the learning transfers to other material that has a similar pattern within it.

Experimental psychology has applications, whether or not we pay attention to them. I fear that the theory of coding may, in subtle ways, lead us and any practitioners who listen to us to think of coding as a natural and desirable form of learning. They would then seek educational reforms mainly by working on the learner. trying to teach him an all-purpose code into which he can transform strange new information. But, if I am right. codes are used only on nonsensical material, and educational reform should take the form of reducing the nonsensical component in the materials being taught and of teaching children to find the patterns in the world around them.

As a prediction about research, I believe that if we can successfully move learning theory from the nonsense syllable to languages and from lists to passages of material, we shall find the concept of "coding" becomes less and less important and interesting. Although the idea of coding is an important advance over the theory of item by item association, we should remember that such an advance is only a step toward the truth, and one must be as willing to pick up the rear foot as he was eager to plant it, ahead, one step ago.

\section{REFERENCES}

Bruner, J. S. The course of cognitive growth. American Psychologist, 1964, 19, 1-15.

Gibson, E. J. Principles of perceptual learning and development. New York: Appleton-Century-Crofts. 1969.

Glanzer, M., \& Clark, W. H. The verbal loop hypothesis: Binary numbers. Journal of Verbal Learning \& Verbal Behavior, 1963, 2, 301-309.

Hubel, D. H., \& Wiesel, T. N. Receptive fields of single neurons in the cat's striate cortex. Journal of Physiology, 1959. 148, 574-591.

Kintsch, W. Learning, memory, and conceptual processes. New York: Wiley, 1970.

Levin, H., \& Turner, E. A. Sentence structure and eye-voice span. Project Literary Reports, September 1966, No. 7. 79-87.

Liberman, A. M., Cooper, F. S., Shankweiler, D. P., \& Studdert-Kennedy, M. Perception of the speech code. Psychological Review, 1967, 74, 431.461.

Posner, M. I., \& Mitchell, R. F. Chronometric analysis of classification. Psychological Review, 1967. 74, 392-409.

Restle, F. Theory of serial pattern learning: Structural treees. Psychological Review, 1970, 77, 481-495.

Restle, F., \& Brown, E. R. Serial pattern learning. Journal of Experimental Psychology, 1970, 83, 120-125.

Restle, F., \& Burnside, B. L. Tracking of serial patterns. Journal of Experimental Psychology, 1972, 95, 299-307.

Restle, F., \& Greeno, J. G. Introduction to mathematical psychology, Reading, Mass: Addison-Wesley, 1970.

Stevens, K. N., \& Halle, M. Remarks on analysis by synthesis and distinctive features. In W. Wathen-Dunn (Ed.), Models for the perception of speech and form. Cambridge. Mass: M.I.T. Press, 1967.

Vitz, P. C., \& Todd, T. C. A coded element model of the perceptual processing of sequential stimuli. Psychological Review, 1969, 76, 433-449.

(Received for publication A ugust 24, 1973.) 